\title{
Pharmacokinetics and Pharmacodynamics of Emicizumab in Persons with Hemophilia A with Factor VIII Inhibitors: HAVEN 1 Study
}

\author{
Christophe Schmitt $^{1}$ Joanne I. Adamkewicz ${ }^{2}$ jin $\mathrm{Xu}^{3} \quad$ Claire Petry $^{1}$ Olivier Catalani ${ }^{4}$ Guy Young $^{5}$ \\ Claude Negrier $^{6}$ Michael U. Callaghan ${ }^{7}$ Gallia G. Levy ${ }^{8}$
}

${ }^{1}$ Department of Clinical Pharmacology, F. Hoffmann-La Roche Ltd, Basel, Switzerland

2 Department of Oncology Biomarker Development, Genentech, Inc., South San Francisco, California, United States

${ }^{3}$ Department of Clinical Research, Genentech, Inc., South San Francisco, California, United States

${ }^{4}$ Department of Pharma-Development, F. Hoffmann-La Roche Ltd, Basel, Switzerland

${ }^{5}$ Hemostasis and Thrombosis Program, Children's Hospital Los Angeles, University of Southern California Keck School of Medicine, Los Angeles, California, United States

${ }^{6}$ Hematology Department, Louis Pradel Hospital, University Claude Bernard, Lyon, France

7 Division of Hematology/Oncology, Children's Hospital of Michigan, Detroit, Michigan, United States

${ }^{8}$ Department of Pharma Development, Genentech, Inc., South San Francisco, California, United States

Thromb Haemost 2021:121:351-360.
Address for correspondence Christophe Schmitt, PharmD, Department of Clinical Pharmacology, F. Hoffmann-La Roche Ltd, Basel CH-4070, Switzerland (e-mail: christophe.schmitt@roche.com).

\section{Abstract}

\section{Keywords}

- emicizumab

- hemophilia A

- pharmacokinetics

- pharmacodynamics
Emicizumab, a bispecific monoclonal antibody, bridges activated factor IX (FIXa) and $F X$, replacing the function of missing FVIIla to restore effective hemostasis in persons with hemophilia A (PwHA). Here we assess pharmacokinetic (PK) and pharmacodynamic (PD) biomarkers in PwHA with FVIII inhibitors in the Phase III HAVEN 1 study (NCT02622321). Blood samples from 112 PwHA receiving $1.5 \mathrm{mg} / \mathrm{kg}$ once-weekly subcutaneous emicizumab were analyzed at central laboratories. Emicizumab concentrations for PK analysis were measured via validated immunoassay. PD effects were assessed using FVIII chromogenic activity assay containing human factors (Hyphen Biophen FVIII:C), and by FXla-triggered thrombin generation (TG). Activated partial thromboplastin time (aPTT), prothrombin time (PT), antigen levels of FIX and FX, fibrinogen, D-dimer, and prothrombin fragment 1.2 (PF1.2) levels were determined. Emicizumab trough concentrations $\geq 50 \mu \mathrm{g} / \mathrm{mL}$ were maintained throughout the study. FVIII-like activity and TG (peak height) correlated with emicizumab concentrations and remained above $20 \mathrm{U} / \mathrm{dL}$ and $100 \mathrm{nM}$, respectively, with a weekly maintenance dose, theoretically converting persons with severe hemophilia $\mathrm{A}$ to a mild disease phenotype. aPTT was normalized at subtherapeutic concentrations of received

April 23, 2020

accepted after revision

August 18, 2020

published online

October 21, 2020
DOI https://doi.org/ $10.1055 / \mathrm{s}-0040-1717114$ ISSN 0340-6245. (c) 2020. The Author(s).

This is an open access article published by Thieme under the terms of the Creative Commons Attribution-NonDerivative-NonCommercial-License, permitting copying and reproduction so long as the original work is given appropriate credit. Contents may not be used for commercial purposes, or adapted, remixed, transformed or built upon. (https://creativecommons.org/ licenses/by-nc-nd/4.0/) Georg Thieme Verlag KG, Rüdigerstraße 14, 70469 Stuttgart, Germany 
emicizumab. Plasma concentrations of target antigens FIX and FX were not significantly affected by emicizumab treatment; nor were fibrinogen, PT (international normalized ratio), D-dimer, or PF1.2. The PK profile of once-weekly emicizumab in HAVEN 1 provides sustained therapeutic plasma levels, consistent with population PK models. Both the PK profile and the PD and safety biomarkers are consistent with the established efficacy of emicizumab prophylaxis in PwHA with FVIII inhibitors.

\section{Introduction}

Hemophilia A results from congenital deficiency of coagulation factor (F) VIII. ${ }^{1}$ Persons with hemophilia A (PwHA) can experience frequent clinical bleeding-related symptoms including easy bruising, prolonged bleeding after trauma or surgery, and spontaneous bleeding into joints, muscles, or soft tissues.

The current standard of care for PwHA with a frequent bleeding phenotype (mostly severe hemophilia) is regular prophylactic intravenous infusions of FVIII, ${ }^{1,2}$ the goal being to maintain target trough FVIII activity levels of $\geq 1 \mathrm{U} / \mathrm{dL}$ to prevent bleeds and mitigate long-term secondary complications. Approximately 30\% of PwHA develop neutralizing alloantibodies (FVIII inhibitors), which render FVIII replacement therapy ineffective. ${ }^{1}$ Prior to the availability of emicizumab, hemostatic treatments for PwHA with FVIII inhibitors were prothrombotic coagulation factors that bypass FVIII. However, bypassing agents (BPAs) such as activated prothrombin complex concentrate (aPCC) and recombinant-activated human FVII (rFVIIa) have suboptimal hemostatic effects and a high treatment burden associated with significant limitations (short half-life, slow intravenous infusion rate). ${ }^{3,4}$

Emicizumab (HEMLIBRA ${ }^{\circledR} ;$ F. Hoffmann-La Roche Ltd, Basel, Switzerland) is a bispecific, humanized, monoclonal antibody that bridges activated FIX (FIXa) and FX, mimicking the cofactor function of missing activated FVIII (FVIIIa), to restore effective hemostasis in PwHA. ${ }^{5,6}$ It has no sequence homology with FVIII, and is therefore unlikely to induce FVIII inhibitors and is unaffected by their presence. ${ }^{5,7}$ Emicizumab has high subcutaneous bioavailability ${ }^{8}$ and a half-life of approximately 30 days, ${ }^{9}$ enabling treatment with once weekly, ${ }^{10}$ every 2 week, ${ }^{11}$ or every 4 week $^{12}$ subcutaneous dosing regimens, thus avoiding the need for frequent intravenous administration.

Following the results of HAVEN $1^{10}$ and HAVEN 2, ${ }^{13,14}$ $1.5 \mathrm{mg} / \mathrm{kg}$ subcutaneous once-weekly emicizumab was approved as a prophylactic treatment for PwHA with FVIII inhibitors of all age groups in several countries (including European Union member states). The original indication and dosing of emicizumab has now been expanded in many countries based on the results of HAVEN $3^{11}$ and HAVEN $4^{12}$ to include $1.5 \mathrm{mg} / \mathrm{kg}$ once weekly, $3.0 \mathrm{mg} / \mathrm{kg}$ every 2 week, or $6.0 \mathrm{mg} / \mathrm{kg}$ every 4 week prophylaxis for PwHA regardless of their inhibitor status. The European Medicines Agency has approved emicizumab for use in patients without FVIII inhibitors only for those with severe $(<1 \mathrm{U} / \mathrm{dL}$ FVIII activity) hemophilia A. ${ }^{15,16}$

HAVEN $1^{10}$ was a pivotal Phase III study designed to evaluate the efficacy, safety, and pharmacokinetics (PK) of subcutaneous once-weekly emicizumab prophylaxis versus no prophylaxis in adult and adolescent (aged $\geq 12$ years) PwHA with FVIII inhibitors. Emicizumab was well tolerated and demonstrated an $87 \%$ reduction in treated bleed annualized bleeding rate (ABR) versus no prophylaxis (ABR [95\% confidence interval [CI]] 2.9 [1.69-5.02] vs. 23.3 [12.33-43.89]). Of those treated with emicizumab, 62.9\% experienced zero treated bleeds. Emicizumab improves upon current treatment options and fulfills a previously unmet medical need. ${ }^{11,12,17,18}$

While emicizumab mimics FVIII cofactor activity, it has fundamental differences from FVIII in terms of PK and biochemical and pharmacological properties. ${ }^{19}$ This article presents the secondary objectives assessing PK, pharmacodynamic (PD), and safety biomarkers from HAVEN 1.

\section{Methods}

\section{Study Design and Patients}

\section{Patients}

Adult and adolescent ( $\geq 12$ years old) PwHA with FVIII inhibitors previously treated with BPAs with suboptimal success were enrolled based on a comprehensive list of inclusion and exclusion criteria. ${ }^{10}$

Participants $(n=113)$ were enrolled between November 18 , 2015 and September 28, 2016; the clinical cut-off date for this analysis was September 8, 2017. All participants provided written informed consent prior to study entry. The study protocol was approved by the relevant independent ethics committee/institutional review board at each participating institution and was conducted in accordance with the principles of the Declaration of Helsinki and Good Clinical Practice.

\section{Study Design}

HAVEN 1, a Phase III, open-label, multicenter, randomized study in PwHA with FVIII inhibitors, took place at 43 centers across 14 countries. Full methods of the HAVEN 1 study have been published previously. ${ }^{10}$ Briefly, participants receiving episodic BPA treatment before study entry were randomized 2:1 to receive either emicizumab prophylaxis ( $\operatorname{arm} A$ ) or no prophylaxis (arm B). Participants previously treated with prophylactic BPAs were assigned to arm $\mathrm{C}$ to receive emicizumab prophylaxis. Participants from a noninterventional study (NCT02476942) ${ }^{20}$ who were unable to enroll in arms A, $B$, or $C$ before enrollment was closed were eligible for arm $D$.

PwHA in arms A, C, and D were given a loading dose of subcutaneous emicizumab $3 \mathrm{mg} / \mathrm{kg}$ once weekly for 4 weeks, followed by $1.5 \mathrm{mg} / \mathrm{kg}$ subcutaneous once weekly maintenance thereafter; PwHA in arm B received no prophylaxis. After completing $\geq 24$ weeks in the study, arm B participants 
could receive emicizumab prophylaxis as described (i.e., $3 \mathrm{mg} / \mathrm{kg}$ once weekly for 4 weeks, $1.5 \mathrm{mg} / \mathrm{kg}$ once weekly thereafter). All participants could receive episodic BPAs for the treatment of breakthrough bleeding, as needed. In case of suboptimal efficacy after $\geq 6$ months of emicizumab prophylaxis, uptitration to $3 \mathrm{mg} / \mathrm{kg}$ once weekly was permitted. ${ }^{10}$ Approved subcutaneous administration sites were the abdomen, upper arm, and thigh.

\section{Blood Sampling and Analyses}

Blood samples for PK and PD analyses were taken from all participants at the following scheduled time points: before first emicizumab dose; immediately prior to emicizumab injection (at trough) every week for the first month; every 2 weeks for the second month; every 4 weeks from the third to the sixth month; every 8 weeks from the seventh to the twelfth month; and every 12 weeks thereafter. Following thrombotic microangiopathy and thromboembolism in two participants treated with an average cumulative aPCC dose of $\geq 100 \mathrm{U} / \mathrm{kg} /$ 24 hours while receiving emicizumab prophylaxis, the HAVEN 1 protocol was amended to recommend the optional collection of additional samples for platelet count, D-dimer, prothrombin fragment 1.2 (PF1.2), and fibrinogen analysis within 24 hours of BPA use from October 2016 onwards.

Blood samples $(2 \mathrm{~mL})$ for emicizumab measurement were collected using ethylenediaminetetraacetic acid-containing plastic tubes. Samples were centrifuged $\left(1,500 \times \mathrm{g}, 4^{\circ} \mathrm{C}\right)$ for 15 minutes to collect plasma and were stored below $-70^{\circ} \mathrm{C}$ prior to analysis.

Blood samples (5.4-18.9 mL depending on body weight) were collected in plastic tubes containing $3.2 \%$ sodium citrate for analysis of: FVIII activity, thrombin generation (TG), activated partial thromboplastin time (aPTT), prothrombin time (PT), FIX and FX antigens, D-dimer, PF1.2, fibrinogen, and von Willebrand factor antigen (VWF:Ag). Samples were centrifuged $(3,000 \times g$, room temperature) for 20 minutes to obtain plasma and samples were stored below $-70^{\circ} \mathrm{C}$ prior to analysis.

Emicizumab plasma concentrations were determined using a validated enzyme-linked immunosorbent assay, performed by QPS Netherlands B.V. (Groningen, The Netherlands). The lower limit of quantitation was $100 \mathrm{ng} / \mathrm{mL}$ in human plasma. Assay precision and accuracy were 9.5 to $13.3 \%$ and 97.6 to $103 \%$, respectively.

All biomarkers (except platelet count) were analyzed at Medpace Reference Laboratories (Cincinnati, Ohio, United States). FVIII activity of emicizumab was measured using a validated chromogenic assay containing human FIXa and FX (Hyphen Biomed, Neuville-sur-Oise, France) with two different calibration curves: high, for samples with $\geq 10 \mathrm{U} / \mathrm{dL}$ FVIII activity; and low, for samples with $<10 \mathrm{U} / \mathrm{dL}$ FVIII activity. Human-derived factors were used in the chromogenic assays as bovine-derived components are insensitive to emicizumab, and therefore cannot be used to measure emicizumab activity. ${ }^{21}$ Of note, FVIII activity reported for PwHA treated with emicizumab with this assay cannot be compared with, or interpreted as equivalent to, FVIII activity reported in participants treated with FVIII; it will, therefore, be called FVIII-like activity throughout the rest of the article. TG was measured with the Calibrated Automated Thrombogram method (Diagnostica Stago, Asnièressur-Seine, France), which used a triggering reagent containing FXIa, as this has been shown to be more robust and sensitive than tissue factor, especially for those with low apparent FVIII activities, as would be expected in HAVEN 1 participants $\left(0-30 \mathrm{U} / \mathrm{dL}\right.$ FVIII). ${ }^{22}$ TG (peak height) was derived from the thrombogram. Clotting times (aPTT and PT), D-dimer, PF1.2, fibrinogen, and VWF:Ag were analyzed using commercial test kits approved for in vitro diagnostic use according to the manufacturers' kit inserts (see Supplementary Material, available in the online version). Protein levels of both FIX and FX were measured using validated immunoassays (Assaypro, St. Charles, Missouri, United States). Platelet count was measured at the local clinical sites as part of regular safety monitoring and was recommended to be measured within 24 hours of BPA administration.

\section{Pharmacokinetics/Pharmacodynamics Analysis}

Exploratory graphical analyses were performed to investigate the PK/PD relationship between emicizumab concentration and FVIII-like activity, TG, or aPTT. The data from all participants were pooled for analysis. With only trough samples, hysteresis could not be checked in the present study. However, as indicated in previous studies in healthy subjects ${ }^{23}$ and in PwHA, ${ }^{6}$ PD markers (e.g., aPTT or TG) are directly linked to emicizumab concentration without time delay (i.e., no hysteresis). Therefore, a direct relationship between PK and PD effect was considered.

The PK/PD relationships for TG and FVIII-like activity could not be appropriately described by a maximum effect $\left(E_{\max }\right)$ model due to the limited range of emicizumab concentrations achieved in the HAVEN 1 study. Linear regressions were, therefore, performed on the "linear" portion of the $E_{\max }$ model (i.e., for concentrations up to $80 \mu \mathrm{g} / \mathrm{mL}$ ).

For aPTT, different inhibitory $E_{\max }$ models were tested for best fit, and an appropriate model was selected on the basis of the Akaike information criterion. Model diagnosis was also performed by visual analysis of the weighted residual plots and by observation of the relative standard error of the estimated variables. The estimates and associated relative standard error were reported. The relationships between emicizumab plasma concentration and aPTT were well described by an inhibitory $E_{\max }$ model:

$$
E=E_{0}-\frac{I_{\max }{ }^{*} C}{C+\mathrm{IC}_{50}}
$$

Here, $E$ is the aPTT, $E_{0}$ is the aPTT at baseline (seconds), $I_{\max }$ is the maximum inhibition at infinite emicizumab concentration, $C$ is the emicizumab plasma concentration $(\mu \mathrm{g} / \mathrm{mL})$, and $\mathrm{IC}_{50}$ is the emicizumab concentration causing half of the maximum effect $(\mu \mathrm{g} / \mathrm{mL})$ ( - Supplementary Table S1, available in the online version).

Graphical investigations of the potential effects of average FIX and FX concentrations (emicizumab target antigens) on the PK/PD relationships were also performed. 
Of note, investigation of the relationship between emicizumab exposure and bleeding events is the subject of dedicated publications. ${ }^{9,24,25}$

\section{Statistical Analysis}

The total HAVEN 1 sample size was based on clinical rather than statistical considerations, taking into account the limited number of PwHA with FVIII inhibitors available for participation, and to collect sufficient data to assess the safety and efficacy of emicizumab as previously described. ${ }^{10}$

All participants initially received the same emicizumab dosing regimen. Consequently, PK and PD data are presented as a single-dose group. Since participants in arm B switched to emicizumab prophylaxis after completing 24 weeks on study, their scheduled time relative to first emicizumab dose was used for graphical displays. PK and PD data were subject to descriptive analysis. Data from participants who uptitrated to $3 \mathrm{mg} / \mathrm{kg}$ once-weekly emicizumab were included in the descriptive statistics until uptitration, and continued to be included in the PK/PD relationship plots after uptitration. PD data in the form of aPTT and TG from participants who had their blood samples drawn via ports and for whom contamination with heparin was suspected were excluded from the summary statistics calculations.

\section{Data Sharing Statement}

Data sharing statement: Qualified researchers may request access to individual patient-level data through the clinical study data request platform (www.clinicalstudydatarequest. com). Further details on Roche's criteria for eligible studies are available at https://clinicalstudydatarequest.com/Study-Sponsors/Study-Sponsors-Roche.aspx. For further details on Roche's Global Policy on the Sharing of Clinical Information and how to request access to related clinical study documents, visit https://www.roche.com/research_and_development/who_we_are_how_we_work/clinical_trials/our_commitment_to_data_sharing. htm.

\section{Results}

\section{Study Population}

A full description of participants enrolled in the HAVEN 1 study, together with baseline demographics and clinical characteristics of each treatment arm, has been published previously. ${ }^{10}$ Briefly, 113 male participants with a median age of 29 years (range: $12-75$ years) were enrolled in HAVEN 1 ; all participants had a historic FVIII inhibitor titer $\geq 5$ Bethesda Units per $\mathrm{mL}(\mathrm{BU} / \mathrm{mL}$ ) (range: 5-5,000 $\mathrm{BU} / \mathrm{mL}$ ), 106 (93.8\%) had a diagnosis of severe congenital hemophilia A, and $69.6 \%$ (78 of 112 ) had target joints. The median duration of exposure to emicizumab was 60.5 weeks (range: 3.3-94.2 weeks). For this analysis, since the primary analysis clinical cut-off date (October 25,2016$)^{10}$ the five outstanding participants in arm B were switched to emicizumab prophylaxis and are now included in the analysis population, and four additional participants have enrolled in arm D. One participant withdrew from the study without receiving any emicizumab treatment, and five participants were withdrawn from emicizumab treatment; three due to adverse events and two following physician or patient decision (-Supplementary Fig. S1, available in the online version).

\section{Pharmacokinetics}

At clinical data cut-off, 112 PwHA who had received at least one emicizumab dose and had at least one postdose emicizumab concentration sample were included in the PK analysis. Mean emicizumab trough concentration $\left(C_{\text {trough }}\right)$ increased with once weekly subcutaneous doses of $3 \mathrm{mg} / \mathrm{kg}$ emicizumab (loading dose); by the end of the loading dose period (week 5), a mean $C_{\text {trough }}$ of $54.1 \mu \mathrm{g} / \mathrm{mL}$ (median [interquartile range [IQR, 25th and 75th percentiles]]: 52.7 [44.5-62.8] $\mu \mathrm{g} / \mathrm{mL}$ ) was achieved ( - Fig. 1). During the maintenance dose phase, $C_{\text {trough }}$ was sustained slightly above $50 \mu \mathrm{g} / \mathrm{mL}$ with $1.5 \mathrm{mg} / \mathrm{kg}$ once weekly subcutaneous emicizumab. The data variability was moderate, with an IQR of 41.6 to $61.61 \mu \mathrm{g} / \mathrm{mL}$.

\section{Pharmacodynamics}

\section{FVIII-Like Chromogenic Activity}

As expected in participants with FVIII deficiency, there was no detectable FVIII activity in PwHA at baseline (-Fig. 2A), except for one participant with mild hemophilia who presented with $15 \mathrm{U} / \mathrm{dL}$ FVIII-like activity at baseline. The participant, who had a highest historical FVIII inhibitor titer of $18 \mathrm{BU} / \mathrm{mL}$, presented with a baseline FVIII inhibitor titer of $17 \mathrm{BU} / \mathrm{mL}$ and lacked documented coagulation factor use immediately prior to sampling. This participant, who has a missense mutation of the $F 8$ gene (Arg2150His), may have developed alloantibodies against exogenous (wild-type) FVIII, but not against his own mutated, dysfunctional, endogenous FVIII, as previously reported with this mutation. ${ }^{26}$

As measured using a validated chromogenic assay containing human FIXa and FX, mean FVIII-like activity increased to $29.8 \mathrm{U} / \mathrm{dL}(95 \% \mathrm{CI}, 15.2-44.3)$ among the study population ( $n=112$ ) at the end of the emicizumab loading dose period, and stabilized above $20 \mathrm{U} / \mathrm{dL}$ thereafter; at week 72, mean FVIII-like activity was $20.3 \mathrm{U} / \mathrm{dL}$ (95\% CI, 12.3-28.4).

\section{Thrombin Generation}

As expected for PwHA with high FVIII inhibitor titers, there was no detectable TG at baseline (-Fig. 2B), except for one participant in whom a peak height of $165 \mathrm{nM}$ was reported (the same participant with a history of mild hemophilia who exhibited $15 \mathrm{U} / \mathrm{dL}$ FVIII-like activity at baseline).

Following the emicizumab loading dose phase, mean TG peak height increased on average among the study population to $108.8 \mathrm{nM}$ (95\% CI, 29.7-187.9) and was sustained thereafter; at week 72 , mean TG peak height was $108.7 \mathrm{nM}$ (95\% CI, 46.3-171.1).

Of note, six participants had no detectable TG at some or all time points despite treatment with emicizumab. In these participants, blood samples were drawn via a port, and contamination with heparin was later confirmed. These blood samples did exhibit chromogenic FVIII-like activity 


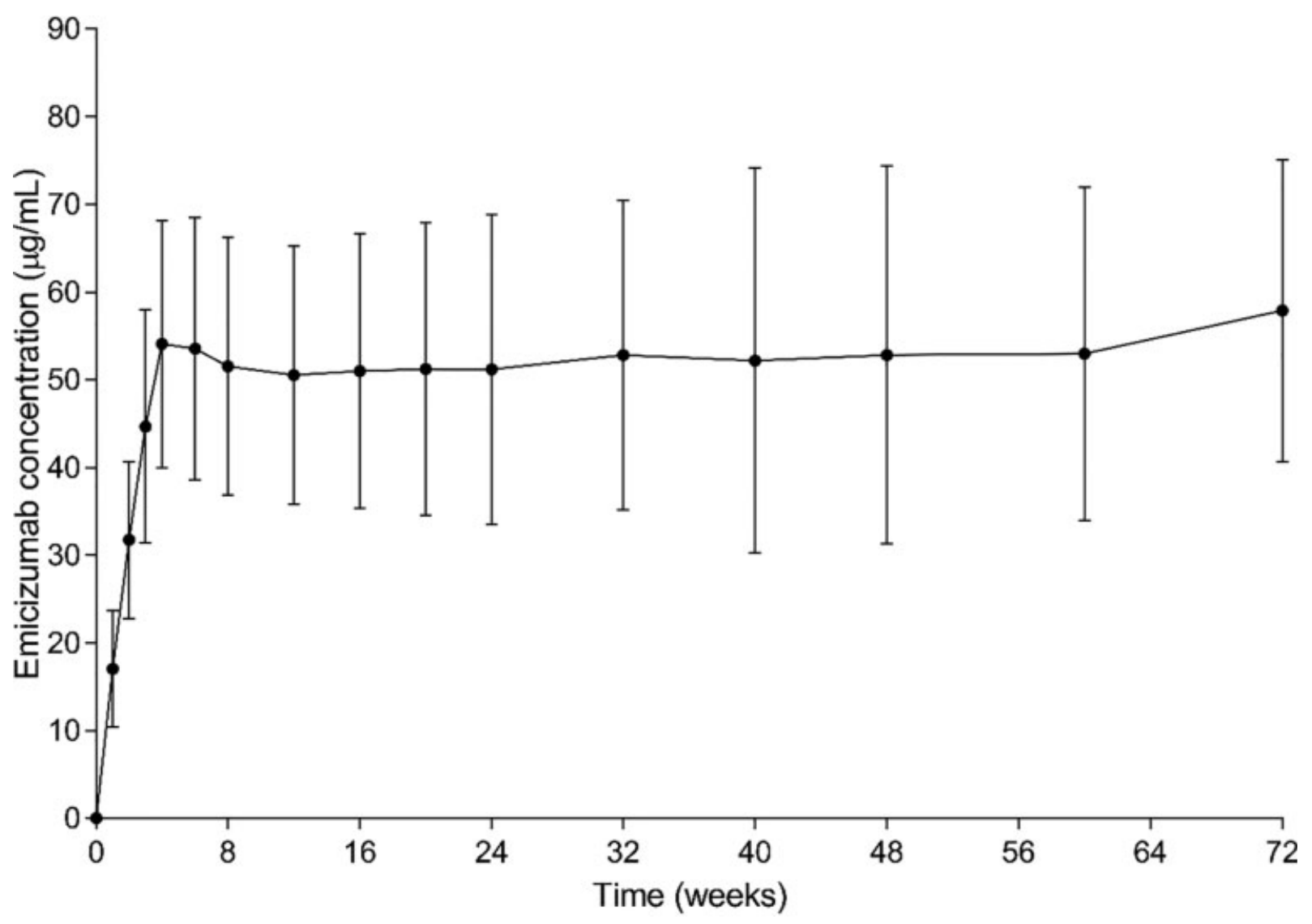

Fig. 1 Trough plasma concentration of emicizumab (mean \pm standard deviation) after 4 loading doses of $3 \mathrm{mg} / \mathrm{kg}$ once-weekly (QW) subcutaneous (SC) for 4 weeks, followed by maintenance dosing of $1.5 \mathrm{mg} / \mathrm{kg}$ QW SC. Means were derived from the values of participants of all emicizumab-treated arms $(n=112)$. For patients in arm B $(n=18)$ who switched to emicizumab prophylaxis after 24 weeks, scheduled time relative to first emicizumab dose was used for graphical displays.

consistent with the patients' emicizumab concentration and the overall population PD data.

\section{Activated Partial Thromboplastin Time}

aPTT was prolonged in all participants at baseline, but was normalized below 30 seconds after the first dose of emicizumab and remained largely within normal limits (laboratory-determined reference range, $23.9-40$ seconds) for the entire duration of treatment (-Fig. 2C); at week 72, mean (standard deviation) aPTT was 23.6 seconds (2.0). A few participants consistently had values just under the lower limit of the normal range. Six participants presented with some prolonged aPTT values despite treatment with emicizumab. These participants (who also had undetectable TG) had their blood samples drawn via a port, and contamination with heparin was suspected.

\section{Prothrombin Time}

Overall, treatment with emicizumab had no effect on PT (international normalized ratio [INR]); the majority of participants had PT between 13 and 14 seconds at baseline (corresponding to INR of 0.9-1.1) and at all subsequent visits while receiving emicizumab once weekly subcutaneously (-Fig. 2D). A minor increase of 0.6 seconds, however, could be seen after the first dose of emicizumab.

\section{Exploratory Safety Biomarkers}

Treatment with emicizumab had no clinically significant effects on plasma concentrations of the target antigens of emicizumab, FIX (-Fig. 3A) and FX (-Fig. 3B). A few elevated values of both FIX and FX plasma concentrations were observed at baseline; these were mainly observed in participants in arm C, who had used prophylactic aPCC before entering the study. Likewise, emicizumab treatment had no clinically significant effects on D-dimer ( - Fig. 3C) or PF1.2 (-Fig. 3D). Highly elevated D-dimer concentrations were observed on single visits in two participants who had used aPCC for the treatment of breakthrough bleeding in the 24 to 48 hours before sample collection. Isolated instances of elevated PF1.2 concentrations were also noted in a few participants, most of whom had used aPCC for treating breakthrough bleeds in the days before sample collection.

Other safety markers that were measured included platelet count (-Supplementary Fig. S2A, available in the online version), fibrinogen (-Supplementary Fig. S2B, available in the online version), and VWF:Ag (-Supplementary Fig. S2C, available in the online version), all of which remained constant within expected limits from screening through to the clinical cut-off. Further safety results have been reported previously. ${ }^{10}$ 

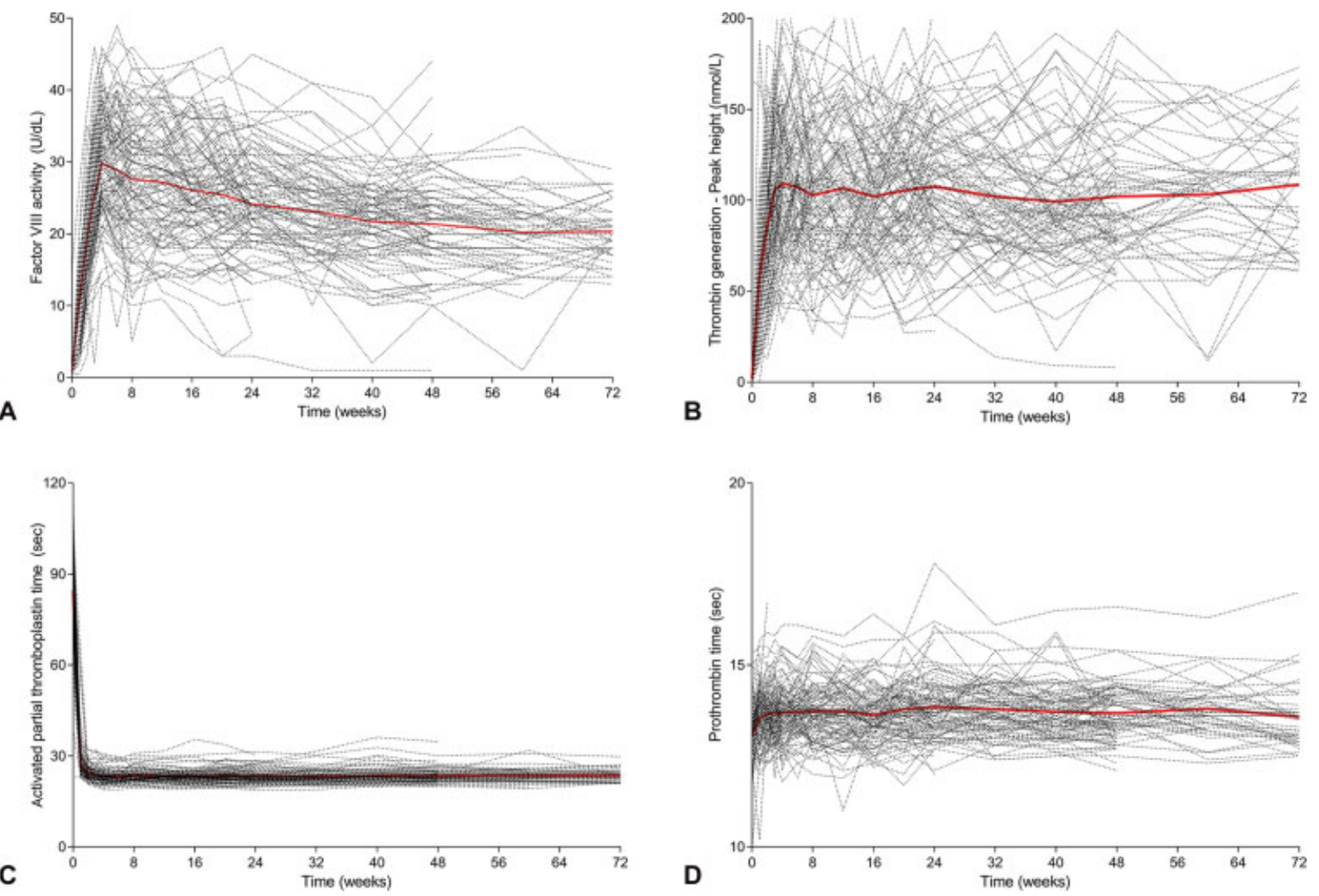

Fig. 2 Pharmacodynamic markers following once-weekly dosing with emicizumab. Chromogenic FVIII-like activity (A), peak height of thrombin generation (TG) (B), activated partial thromboplastin time (aPTT) (C), and prothrombin time (PT) (D). Dashed lines: individual profiles; red line: mean profile. ( $n=112$ for FVIII-like activity and PT; $n=102$ for TG and aPTT).

\section{Pharmacokinetic/Pharmacodynamic Relationships} Chromogenic FVIII-like activity (measured using a FVIII chromogenic assay with human FIXa and FX) was well correlated with emicizumab plasma concentration (-Fig. 4 A). It increased linearly up to approximately 80 to $100 \mu \mathrm{g} / \mathrm{mL}$ and seemed to flatten thereafter, although the scarcity of data values above $100 \mu \mathrm{g} / \mathrm{mL}$ emicizumab prevented complete characterization in this range. Similar to FVIII-like activity, TG (peak height) also correlated well with emicizumab levels in an apparently linear fashion up to emicizumab concentrations of approximately 80 to $100 \mu \mathrm{g} / \mathrm{mL}$ ( - Fig. 4B). Of note, the concentration of FIX or FX did not appear to impact the PK/PD relationships of either FVIII-like activity or TG (-Supplementary Figs. $\mathbf{3 3}$ and $\mathbf{5 4}$, available in the online version). A concentration-dependent normalization of aPTT ( $<40$ seconds) was observed starting at plasma emicizumab concentrations of $\geq 5 \mu \mathrm{g} / \mathrm{mL}$, with a maximal effect achieved at concentrations $\geq 30 \mu \mathrm{g} / \mathrm{mL}$ ( - Fig. 4C).

\section{Discussion}

Emicizumab is a novel and recently approved therapy for PwHA. Its biochemical and pharmacological properties enable it to address challenges faced by PwHA. Emicizumab allows for subcutaneous rather than intravenous dosing, a more convenient once weekly, every 2 week, or every 4 week administration schedule, functionality as a FVIIla-mimicking cofactor without being affected by the presence of FVIII inhibitors (providing effective bleed control in PwHA both with/without FVIII inhibitors), and lack of induction of FVIII inhibitors. ${ }^{11,23}$

The HAVEN 1 dosing regimen of $3 \mathrm{mg} / \mathrm{kg}$ once weekly emicizumab for 4 weeks followed by $1.5 \mathrm{mg} / \mathrm{kg}$ once weekly emicizumab thereafter was selected by population PK/efficacy modeling $^{9}$ to rapidly achieve and to maintain thereafter therapeutic emicizumab $C_{\text {trough. }}$. At the end of the loading dose period, mean $C_{\text {trough }}$ levels slightly above $50 \mu \mathrm{g} / \mathrm{mL}$ were achieved on average among the study population, and were maintained thereafter with $1.5 \mathrm{mg} / \mathrm{kg}$ once weekly dosing for the entire study duration (> 16 months). Owing to its long elimination half-life of approximately 1 month, ${ }^{23}$ minimal peak-trough fluctuation is expected with emicizumab. ${ }^{9}$ This provided sustained therapeutic exposure to emicizumab for the majority of participants. Based on an estimated potential conversion factor of $0.3 \mathrm{U} / \mathrm{dL}$ of FVIII activity per $\mu \mathrm{g} / \mathrm{mL}$ of emicizumab, ${ }^{6}$ a mean steady-state $C_{\text {trough }}$ of $\geq 50 \mu \mathrm{g} / \mathrm{mL}$ is expected to continuously provide an equivalent FVIII activity of $\geq 15 \mathrm{U} / \mathrm{dL}$. This sustained level of protection, which is considered sufficient to reduce the risk of joint bleeding, ${ }^{27}$ was confirmed by the substantial reduction in individual ABRs for most participants in HAVEN $1 .^{10}$

Activation of FX by emicizumab/FIXa and the subsequent downstream effects on the coagulation cascade were monitored in HAVEN 1 via various PD markers. Unlike FVIII, emicizumab does not require a rate-limiting activation step but instead directly mimics the cofactor activity of FVIIIa. ${ }^{5}$ Therefore, the 

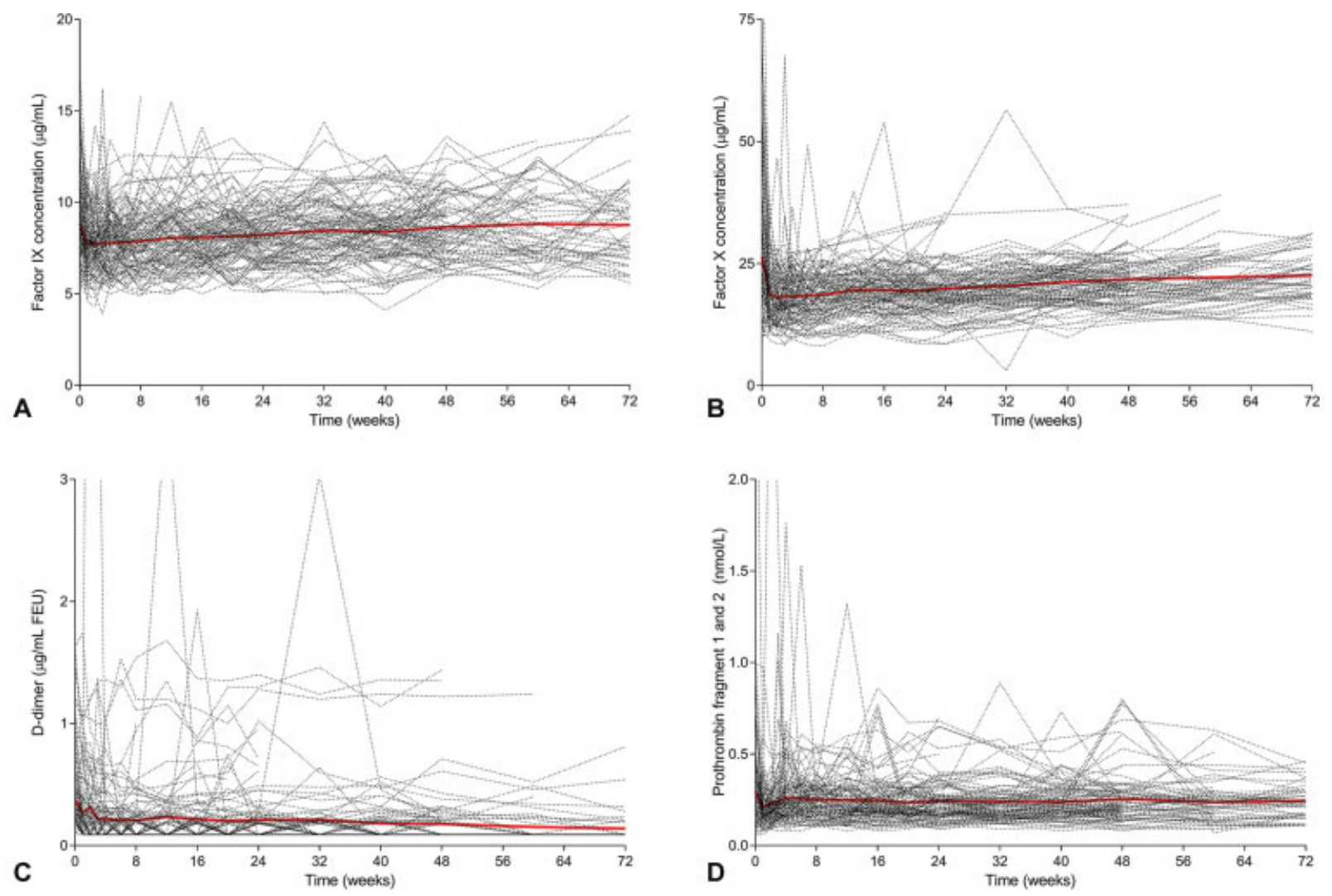

Fig. 3 Safety biomarkers during once-weekly dosing with emicizumab $(n=112)$. FIX antigen (A), FX antigen (B), D-dimer (C), prothrombin fragment 1 and 2 (PF1.2) (D). Dashed lines: individual profiles; red line: mean profile for FIX and FX antigen; median and interquartile range for D-dimer and prothrombin fragment 1 and 2. Upper limit of normal values: D-dimer $(0.5 \mu \mathrm{g} / \mathrm{mL})$; PF1.2 $(1.2 \mathrm{nmol} / \mathrm{L})$.

shortening effect of emicizumab on aPTT is considerably greater than that of FVIII. Consequently, conventional aPTT-based assays (1-stage) that measure FVIII activity are significantly affected by emicizumab and report artificially elevated FVIII activity. ${ }^{28}$ In the present study, the FVIII-like activity of emicizumab was monitored with a chromogenic FVIII activity assay using human FIXa and FX. FVIII-like activity increased with emicizumab loading doses, achieving activity levels of approximately $30 \mathrm{U} / \mathrm{dL}$ at the end of the loading dose period (week 5). With the administration of maintenance emicizumab doses, FVIII-like activity stabilized at approximately $20 \mathrm{U} / \mathrm{dL}$ on average among the study population, corresponding to approximately $20 \%$ of the FVIII-like activity seen in healthy subjects. ${ }^{29}$ The slight apparent decline in FVIII-like activity over time is thought to be the result of a signal drift in the assay (i.e., a change in signal level [FVIII activity] at a given emicizumab concentration with time), likely due to a change in the manufacture of multiple assay kits. This explanation for the artifact in the chromogenic FVIII activity assay was further supported by the absence of a similar decline or difference between groups in either emicizumab plasma concentration or TG.

Chromogenic FVIII-like activity and emicizumab concentration levels were well correlated, with a linear increase in FVIIIlike activity with emicizumab plasma concentration up to 80 $\mu \mathrm{g} / \mathrm{mL}$ ( - Supplementary Fig. S5, available in the online version). Of note, reported FVIII-like activity for PwHA treated with emicizumab should not be interpreted as equivalent to FVIII activity reported in participants treated with FVIII. Due to the different enzymatic cofactor properties of the two molecules, FVIII-like activity can only be used as an approximation of hemostatic emicizumab activity in vivo. ${ }^{5,19}$ Nevertheless, the increase and steady maintenance of FVIII-like activity provides a relative indication of the procoagulant activity of emicizumab.

Similarly, TG (peak height) increased following the initiation of treatment with emicizumab. By the end of the loading dose period, TG peak height of approximately $110 \mathrm{nM}$, equivalent to 20 to $30 \%$ of that in healthy people $(316-488 \mathrm{nM}),{ }^{23}$ was observed and sustained thereafter with emicizumab $1.5 \mathrm{mg} / \mathrm{kg}$ once weekly maintenance doses. TG was well correlated with emicizumab plasma concentration (-Supplementary Fig. 56, available in the online version). This was, however, associated with relatively large variability, possibly due in part to the concomitant use of BPAs for the treatment of breakthrough bleeding; such variability also aligns with data reported for TG values in both PwHA and healthy people. ${ }^{30-32}$ These TG peak height results confirmed the procoagulant effect of emicizumab and demonstrated the ability of TG assays to provide an indication of emicizumab procoagulant activity. Although availability of TG assays is limited to specialized laboratories, this usage was evidenced by a recently published case in which TG was used to guide the management of breakthrough bleeds with BPAs in a participant receiving emicizumab. ${ }^{33}$

Comparison of the measured values of FVIII-like activity and TG peak height in this study with available reference data suggests that the procoagulant activity achieved in HAVEN 1 corresponded to approximately 15 to $30 \%$ of that 
A
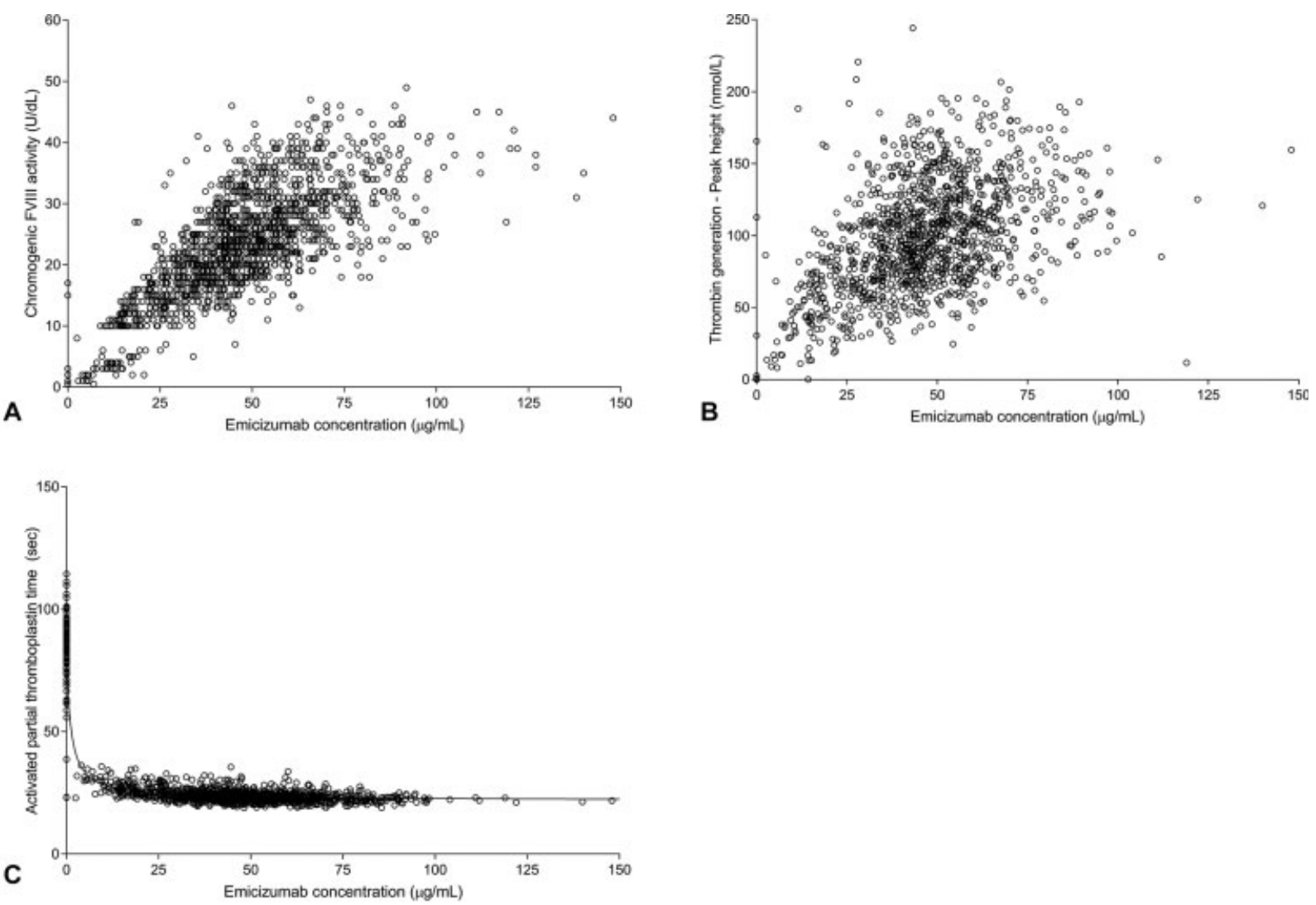

Fig. 4 Pharmacokinetic/pharmacodynamic relationships. Correlation between emicizumab plasma concentrations and chromogenic FVIII-like activity (A), peak height of thrombin generation (B), activated partial thromboplastin time (C). (A) Gap at $10 \mathrm{U} / \mathrm{dL}$ reflects the use of two different calibration curves. (C) Line indicates model.

seen in healthy normo-coagulative people. ${ }^{23,29}$ This suggests that persons with severe hemophilia A treated with emicizumab prophylaxis at a maintenance dose of $1.5 \mathrm{mg} / \mathrm{kg} /$ week could theoretically be converted to a mild hemophilia disease phenotype. Of note, similar findings have recently been obtained with human chromogenic FVIII and TG assays from direct comparisons between healthy adults, PwHA and FVIII inhibitors receiving emicizumab, and persons with mild hemophilia A without FVIII inhibitors receiving no therapy. ${ }^{34}$

FIX and FX, the target antigens of emicizumab, have the potential to impact its procoagulant activity. ${ }^{31}$ However, in the range of concentrations seen in this study (FIX, 6-12 ng/mL; FX, 11-32 ng/mL), FIX and FX concentrations did not affect the PK/PD relationships between emicizumab and FVIII-like activity (- Supplementary Fig. S3, available in the online version) or TG (- Supplementary Fig. S4, available in the online version).

As reported in previous studies, emicizumab had a strong effect on aPTT. ${ }^{6,26}$ aPTT was shortened to within normal limits after the first dose of emicizumab and remained largely within these limits thereafter. The normalization of aPTT at low emicizumab concentrations ( $\geq 5 \mu \mathrm{g} / \mathrm{mL}$ ), with an estimated $\mathrm{IC}_{50}$ of $1.1 \mu \mathrm{g} / \mathrm{mL}$, is thought to be a direct consequence of the mechanism of action of emicizumab (as discussed above) and its resulting interference with clotting time-based assay methods. ${ }^{6}$ Normalization of aPTT occurs at subtherapeutic emicizumab concentrations. ${ }^{9,35,36}$ Consequently, aPTT is not an accurate marker of hemostasis in the presence of emicizumab and should not be used to monitor emicizumab efficacy. However, in the presence of unanticipated bleeding event together with low values of FVIII-like activity, marked increase of aPTT could be an indicator of the presence of anti-emicizumab antibody or noncompliance.

Emicizumab is not expected to affect the extrinsic pathway of the coagulation cascade. However, emicizumab has potential for a modest interference effect on the activation of FX by the FVIIa-TF complex due to the binding of emicizumab to FX and subsequent steric hindrance. ${ }^{37}$ De facto, a tiny increase of PT was noticed after the first dose of emicizumab. This corresponded to approximately 0.05 INR units and was not considered clinically relevant. PT remained stable thereafter throughout the duration of the study.

Owing to its low target-antigen affinity, emicizumab had no effect on FIX or FX concentrations, confirming previous findings. ${ }^{6}$ Slightly elevated FIX and FX concentrations were observed, mainly at baseline (i.e., in the absence of emicizumab); these are believed to reflect previous use of aPCC containing both FIX and $\mathrm{FX}^{38}$ before study entry.

In terms of the safety markers, emicizumab had no effect on D-dimer or PF1.2 levels. Overall, this is consistent with the inability of emicizumab to activate coagulation in the absence of an initiating signal. There were isolated instances in which changes in these markers were observed; however, these appeared to be related to the use of BPAs (aPCC and rFVIIa) 
either before study entry or for treatment of breakthrough bleeding ( - Supplementary Fig. $\mathbf{5 7}$, available in the online version). Furthermore, emicizumab had no effect on platelet count, fibrinogen, or VWF:Ag (- Supplementary Fig. S2, available in the online version). The absence of PT prolongation, platelet count decrease, or fibrinogen decrease in participants treated with emicizumab confirms that emicizumab alone does not induce consumptive coagulopathy. Altogether, these results were aligned with the overall safety profile of emicizumab. ${ }^{10-13}$

In conclusion, the PK profile of $1.5 \mathrm{mg} / \mathrm{kg}$ subcutaneously once weekly emicizumab in HAVEN 1 was consistent with population PK models and provides prophylactic bleed control in a majority of PwHA with FVIII inhibitors. ${ }^{9}$ With regard to PD, both chromogenic FVIII-like activity and TG correlated with emicizumab concentrations and demonstrated stable activity throughout the maintenance-dosing period. Due to the differing biochemical characteristics of FVIII and emicizumab, the chromogenic FVIII assay may only be used to approximate PD effects of emicizumab in PwHA. TG, which provides a global assessment of coagulation, may have potential to confirm the pharmacological effect of emicizumab and guide BPA use in emicizumabtreated PwHA in case of surgeries or breakthrough bleeding. In addition to standardization of the TG assay, further investigations are needed to confirm this conclusion. FIX and FX levels and a panel of coagulation assays and markers including D-dimer were unaffected by emicizumab. aPTT, which is normalized at subtherapeutic emicizumab levels, should not be used to guide clinical decisions regarding PwHA receiving emicizumab. Studies are underway to determine the effects of emicizumab on different assay classes not examined here, to inform appropriate changes in clinical laboratory practice to better accommodate emicizumab. $^{28}$

\section{What is known about this topic?}

- Emicizumab, a humanized, bispecific antibody, mimics missing activated factor VIII (FVIII) function in persons with hemophilia A (PwHA).

- In the HAVEN 1 study, emicizumab administered subcutaneously once a week resulted in a decrease in the annualized bleed rate of PwHA with FVIII inhibitors when compared with on-demand and prophylactic treatment with bypassing agents.

\section{What does this paper add?}

- During HAVEN 1, emicizumab administration provided sustained therapeutic trough plasma concentrations.

- FVIII chromogenic activity and thrombin generation assay provided an indication of emicizumab procoagulant activity.

- Based on these biomarkers, emicizumab may theoretically convert persons with severe hemophilia $\mathrm{A}$ to a mild disease phenotype.

\section{Authors' Contributions}

C.S., J.I.A., C.N., M.U.C., and G.G.L. contributed to the development of the HAVEN 1 study design. C.P. and O.C. contributed to the acquisition of data for these analyses. C.S., J.I.A., J.X., C.P., O.C., G.Y., C.N., M.U.C., and G.G.L. contributed to data analysis and interpretation. All authors critically reviewed this manuscript and approved the final version for submission.

Funding

This study was sponsored by F. Hoffmann-La Roche Ltd.

\section{Conflict of Interest}

C.S., J.I.A., C.P., and G.G.L. are employees of F. Hoffmann-La Roche Ltd/Genentech, Inc. and hold stocks in F. HoffmannLa Roche Ltd. J.X. is an employee of Gilead Sciences, and previous employee of F. Hoffmann-La Roche Ltd/Genentech, Inc. with stocks in F. Hoffmann-La Roche Ltd. O.C. is an employee of F. Hoffmann-La Roche Ltd. G.Y. has received grants and personal fees from Genentech, Inc./F. Hoffmann-La Roche Ltd, Takeda, and Grifols and personal fees from Novo Nordisk, UniQure, Sanofi, Spark Therapeutics, and BioMarin. C.N. has received personal fees from Bayer, CSL Behring, Freeline, LFB, Novo Nordisk, Octapharma, Pfizer, Sanofi, Shire, and Spark Therapeutics; and personal fees and other from Sobi and F. HoffmannLa Roche Ltd. M.U.C. has received personal fees from F. Hoffmann-La Roche Ltd, Genentech, Inc., Bayer, Shire/ Takeda, Pfizer, Novo Nordisk, Bioverative/Sanofi, Global Blood Therapeutics, Spark Therapeutics, BioMarin, Kedrion, Octapharma, Grifols; and has equity in Alnylam.

\section{Acknowledgments}

Medical writing assistance for this manuscript was provided by Sophie Nobes, BSc, of Gardiner-Caldwell Communications, and was funded by F. Hoffmann-La Roche Ltd.

\section{References}

1 Makris M, Kasper C. The World Federation of Hemophilia guideline on management of haemophilia. Haemophilia 2013;19(01):1

2 Valentino LA, Mamonov V, Hellmann A, et al; Prophylaxis Study Group. A randomized comparison of two prophylaxis regimens and a paired comparison of on-demand and prophylaxis treatments in hemophilia A management. J Thromb Haemost 2012;10 (03):359-367

3 Kempton CL, Meeks SL. Toward optimal therapy for inhibitors in hemophilia. Blood 2014;124(23):3365-3372

4 Maahs J, Donkin J, Recht M, Cooper DL. Mixing and administration times of bypassing agents: observations from the Dosing Observational Study in Hemophilia (DOSE). J Blood Med 2014; 5:153-156

5 Kitazawa T, Igawa T, Sampei Z, et al. A bispecific antibody to factors IXa and $\mathrm{X}$ restores factor VIII hemostatic activity in a hemophilia A model. Nat Med 2012;18(10):1570-1574

6 Shima M, Hanabusa H, Taki M, et al. Factor VIII-mimetic function of humanized bispecific antibody in hemophilia A. N Engl J Med 2016;374(21):2044-2053

7 Sampei Z, Igawa T, Soeda T, et al. Identification and multidimensional optimization of an asymmetric bispecific $\operatorname{IgG}$ antibody mimicking the function of factor VIII cofactor activity. PLoS One 2013;8(02):e57479 
8 Kotani N, Yoneyama K, Kawakami N, Shimuta T, Fukase H, Kawanishi T. Relative and absolute bioavailability study of emicizumab to bridge drug products and subcutaneous injection sites in healthy volunteers. Clin Pharmacol Drug Dev 2019;8(06):702-712

9 Yoneyama K, Schmitt C, Kotani N, et al. A pharmacometric approach to substitute for a conventional dose-finding study in rare diseases: example of phase III dose selection for emicizumab in hemophilia A. Clin Pharmacokinet 2018;57(09):1123-1134

10 Oldenburg J, Mahlangu JN, Kim B, et al. Emicizumab prophylaxis in hemophilia A with inhibitors. N Engl J Med 2017;377(09):809-818

11 Mahlangu J, Oldenburg J, Paz-Priel I, et al. Emicizumab prophylaxis in patients who have hemophilia A without inhibitors. N Engl J Med 2018;379(09):811-822

12 Pipe SW, Shima M, Lehle M, et al. Efficacy, safety, and pharmacokinetics of emicizumab prophylaxis given every 4 weeks in people with haemophilia A (HAVEN 4): a multicentre, open-label, nonrandomised phase 3 study. Lancet Haematol 2019;6(06):e295-e305

13 Young G, Liesner R, Sidonio RF, et al. Emicizumab prophylaxis provides flexible and effective bleed control in children with hemophilia A with inhibitors: results from the HAVEN 2 study. 60th Annual Meeting of the American Society of Hematology (ASH); 2018 December 1-4; San Diego, CA2018

14 Young G, Liesner R, Chang T, et al. A multicenter, open-label phase 3 study of emicizumab prophylaxis in children with hemophilia A with inhibitors. Blood 2019;134(24):2127-2138

15 European Medicines Agency. HEMLIBRA $®$ solution for injection: emicizumab pilEa. Initial EU approval; 2018

16 Genentech. HEMLIBRA ${ }^{\circledR}$ (emicizumab-kxwh) injection for subcutaneous use, prescribing information. Revised 10/2018; 2018

17 Lieuw K. Many factor VIII products available in the treatment of hemophilia A: an embarrassment of riches? J Blood Med 2017; 8:67-73

18 Petrini P. Identifying and overcoming barriers to prophylaxis in the management of haemophilia. Haemophilia 2007;13(Suppl 2):16-22

19 Lenting PJ, Denis CV, Christophe OD. Emicizumab, a bispecific antibody recognizing coagulation factors IX and X: how does it actually compare to factor VIII? Blood 2017;130(23):2463-2468

20 Mahlangu J, Oldenburg J, Callaghan MU, et al. Bleeding events and safety outcomes in persons with haemophilia A with inhibitors: a prospective, multi-centre, non-interventional study. Haemophilia 2018;24(06):921-929

21 Calatzis A, Kotani N, Levy GG, Adamkewicz JI. Effect of emicizumab (ACE910) - a humanized bispecific antibody mimicking FVIII cofactor function - on a variety of assay systems. 1st European Congress on Thrombosis and Haemostasis (ECTH); 2016 September 28-30; The Hague, The Netherlands2016

22 Waters EK, Hilden I, Sørensen BB, Ezban M, Holm PK. Thrombin generation assay using factor XIa to measure factors VIII and IX and their glycoPEGylated derivatives is robust and sensitive. J Thromb Haemost 2015;13(11):2041-2052

23 Uchida N, Sambe T, Yoneyama K, et al. A first-in-human phase 1 study of ACE910, a novel factor VIII-mimetic bispecific antibody, in healthy subjects. Blood 2016;127(13):1633-1641

24 Retout S, Schmitt C, Petry C, Mercier F, Frey N. Population pharmacokinetic analysis and exploratory exposure-bleeding rate relation- ship of emicizumab in adult and pediatric persons with hemophilia A. Clin Pharmacokinet 2020. Doi: 10.1007/s40262-020-00904-z

25 Jonsson F, Schmitt C, Petry C, Mercier F, Frey N, Retout S. Exposureresponse modeling of emicizumab for the prophylaxis of bleeding in haemophilia A patients with and without inhibitors against factor VIII. Res Pract Thromb Haemost 2019;3(S1):1-891

26 Peerlinck K, Jacquemin MG, Arnout J, et al. Antifactor VIII antibody inhibiting allogeneic but not autologous factor VIII in patients with mild hemophilia A. Blood 1999;93(07):2267-2273

27 den Uijl IE, Fischer K, Van Der Bom JG, Grobbee DE, Rosendaal FR, Plug I. Analysis of low frequency bleeding data: the association of joint bleeds according to baseline FVIII activity levels. Haemophilia 2011;17(01):41-44

28 Adamkewicz JI, Chen DC, Paz-Priel I. Effects and interferences of emicizumab, a humanised bispecific antibody mimicking activated factor VIII cofactor function, on coagulation assays. Thromb Haemost 2019;119(07):1084-1093

29 Li H, Zhang W, Petry C, et al. Evaluation of the pharmacokinetics, pharmacodynamics, and safety of a single dose of emicizumab in healthy Chinese subjects. Clin Pharmacol Drug Dev 2020. Doi: 10.1002/cpdd. 805

30 Schmitt C, Pannier A, McIntyre C, et al. Crossover dose escalation study to assess safety, pharmacokinetics, and pharmacodynamics of single doses of R1663, an oral factor Xa inhibitor, in healthy male volunteers. J Clin Pharmacol 2012;52(04):499-510

31 van Veen JJ, Gatt A, Bowyer AE, Cooper PC, Kitchen S, Makris M. Calibrated automated thrombin generation and modified thromboelastometry in haemophilia A. Thromb Res 2009;123(06): 895-901

32 Aghighi S, Riddell A, Lee CA, Brown SA, Tuddenham E, Chowdary P. Global coagulation assays in hemophilia A: a comparison to conventional assays. Res Pract Thromb Haemost 2019;4(02):298-308

33 Dargaud Y, Lienhart A, Janbain M, Le Quellec S, Enjolras N, Negrier C. Use of thrombin generation assay to personalize treatment of breakthrough bleeds in a patient with hemophilia and inhibitors receiving prophylaxis with emicizumab. Haematologica 2018; 103(04):e181-e183

34 Manco-Johnson M, Wang M, Boulden Warren B, et al. Emicizumab improves factor $\mathrm{X}$ activation and thrombin generation similar to mild hemophilia in persons with hemophilia $A$ and inhibitors. Res Pract Thromb Haemost 2019;3(S1):336

35 Adamkewicz JI, Schmitt C, Asikanius E, et al. Factor VIII Inhibitor Testing Using a Validated Chromogenic Bethesda Assay (CBA) in HAVEN 1 (bh29884), a Phase 3 Trial of Emicizumab in Persons with Hemophilia A (PwHA) with Inhibitors. Berlin, Germany: International Society on Thrombosis and Haemostasis; 2017

36 Shima M, Hanabusa H, Taki M, et al. Long-term safety and efficacy of emicizumab in a phase $1 / 2$ study in patients with hemophilia A with or without inhibitors. Blood Adv 2017;1(22):1891-1899

37 Kitazawa T, Esaki K, Tachibana T, et al. Factor VIIIa-mimetic cofactor activity of a bispecific antibody to factors IX/IXa and $\mathrm{X} / \mathrm{Xa}$, emicizumab, depends on its ability to bridge the antigens. Thromb Haemost 2017;117(07):1348-1357

38 Food and Drug Administration. FEIBA ${ }^{\circledR}$ for intravenous use, prescribing information. Initial U.S. Approval: 1986; 2013 